\title{
Results of the 2016 EMS User Survey for zbMATH
}

Ingo Brüggemann (Max Planck Institute for for Mathematics in the Sciences, Leipzig, Germany), Klaus Hulek (Gottfried Wilhelm Leibniz Universität Hannover, Germany) and Olaf Teschke (FIZ Karlsruhe, Berlin, Germany)

In 2005, the European Mathematical Society took the initiative to appoint a Scientific User Committee (SCUC) of zbMATH (then Zentralblatt MATH). The main intent was to actively involve the scientific community in a number of developments that were felt to be both necessary and important. Jean-Pierre Bourguignon, who had been a driving force for the creation of the SCUC, also assumed the duties of the first chair. The most urgent issues at the time were the lack of appropriate author identification, the question of use and misuse of reference data and citation measures, and interface functionality. Further developments since then have been the interlinking with open sources like EuDML and arXiv, the database of mathematical software swMATH and formula search.

In 2012, the SCUC (most notably by the efforts of its then chair, Stephan Klaus, from the Oberwolfach Research Institute for Mathematics) prepared a user survey to gather information on the priorities for zbMATH developments from a user perspective. The survey was distributed at the 6th ECM at Kraków on both paper and online forms. Four years later, during the 7ECM at Berlin, a renewed survey was conducted with the main aim of evaluating recent developments but also identifying future directions. At the same time, the results also served as a report on the perception of zbMATH developments under more than a decade of guidance by the SCUC.

While the 7ECM was one of the main dissemination channels for the survey, others (like the zbMATH entry page and reviewer and EMS member mailings) ensured survey participation that reflected diverse usage quite well. Though $66 \%$ of the 209 respondents were from Europe (and 22\% from Germany), indicating a slight conference participation bias, there was also participa- tion of over $13 \%$ from the U.S. and $10 \%$ from South America; Asia was the only large region relatively underrepresented. While more than $60 \%$ of the participants held professorships, there was also broad participation from postdoctoral and doctoral researchers, students and mathematicians holding other positions. Also, the subject areas (according to the MSC) turned out to be as broad as one could reasonably expect.

The first questions were directed to search customs. On a general level, arguably the most significant result is that a majority of mathematicians rely on maths-specific services (arXiv, MathSciNet, zbMATH) in comparison to generic providers like ISI, Scopus and Google Scholar. From a zbMATH perspective, the most positive results are that this service is today used more frequently by $48 \%$ of users compared to five years ago (38\% use it at about the same level and $14 \%$ use it less).

The preferred search topics are quite diverse and often specific: while author is by a slight margin the most preferred aspect, title comes in a close second, while more specific facets like MSC or even formulae are considered relevant by a majority. Even more surprising was the extensive use of free-text feedback for this question. Almost half of all respondents indicated the use of sometimes quite sophisticated combinations of search aspects. The option of extensive logical combinations, which has been additionally supported by filters for some years, is obviously heavily used. Likewise, basically all aspects of the search results (metadata, full text links, reviews, citations, profiles) are considered almost equally important.

Further questions were specifically concerned with new developments. Concerning the zbMATH author database, $52 \%$ of the answers confirm that it has choose the appropriate response for each item:

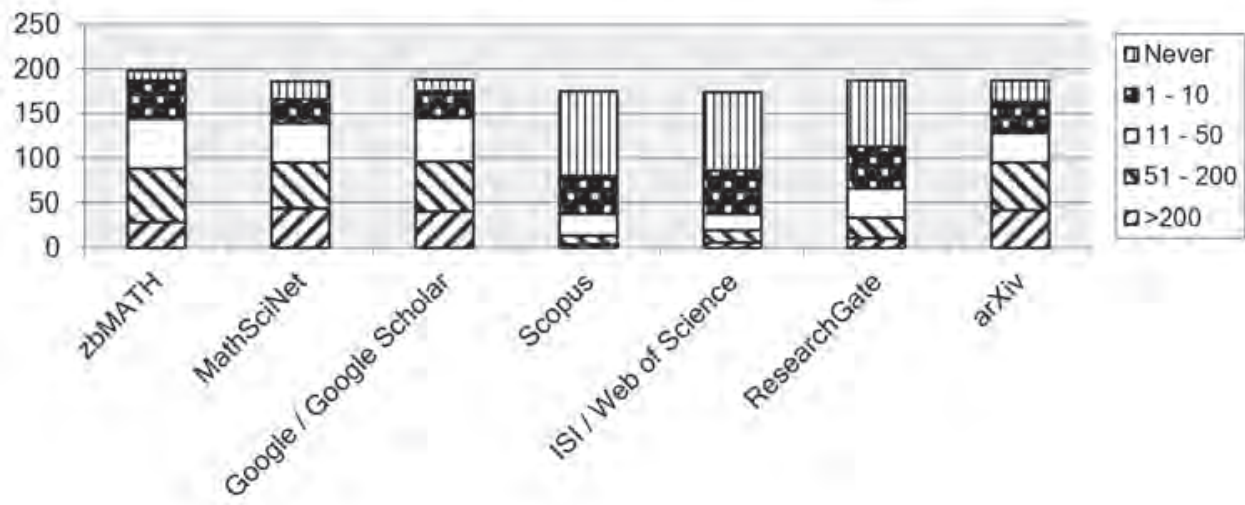

Survey responses concerning search customs of mathematicians. 
improved significantly $(41 \%$ of the participants agree with this statement in principle). On this matter, the quality of the information is, in general, much more highly valued than the quantity - the most important aspects are correct author disambiguation, comprehensive information and clean attribution. More specifically, for example, precise author disambiguation was considered much more important (more than $85 \%$ ) than author citation statistics (30\%). The SCUC also included here a question directly comparing to the MathSciNet author database, which was preferred by $25 \%$, while $22 \%$ found zbMATH more reliable. The majority ( $53 \%$ ) voted that both services have their strengths and weaknesses.

A second large development has been the gradual inclusion of reference data and profiles, which is currently available for a third of recent publications. A difficult aspect here is that this is an ongoing process, and data and profiles are therefore necessarily incomplete. An overwhelming majority $(80 \%)$ understood this and agree with the inclusion of this feature at an early stage, while less than $2 \%$ were against this and would have preferred to omit potentially incomplete citation information. A general fear connected to the integration of reference data into documents and profiles was that this may give quantitative measures an unjustified prevalence over individual quality assessment (as provided by, for example, reviews). The survey does not yet support this - a large majority (about $75 \%$ ) of users use citations to discover other interesting work rather than using them as a tool to assess impact. The general experience that mathematicians are quite aware of the fallacies connected to superficial use of statistics seems to prevail.

Several further functions have been implemented in the service, like filters, profiles, a software database and formula search. The functionality is generally appreciated (with average marks ranging from $3.5 / 5$ for bibtex to $>4 / 5$ for the search function) and $86 \%$ of the respondents think that the service has improved decisively since 2011.

Completeness of entries and quality of reviews are issues for a service like zbMATH that require continued efforts and are naturally always a subject of discussion. While the completeness is generally viewed favourably (4.2/5, with some limitations for very recent articles) and reviews are usually considered as correct (4/5), there is room for improvement regarding the frequency (3.6/5), timeliness (3.7/5) and quality (3.8/5) of reviews, as well as for the suitability of reviewers (3.8/5). Since the decisive factor here is the availability of reviewers, we take the opportunity to encourage the reader to join the reviewer community to facilitate further improvements here. ${ }^{1}$

The question of possible future developments was naturally one of the most interesting ones for us. The diverse answers of fields that were considered relevant left no doubt that there will be an ample amount of work ahead of us in the years to come! User priorities ranged from aspects of historical importance like the digitisation of scans (considered highly desirable with 4/5), gradual improvements like further integration of full text links

\footnotetext{
1 https://zbmath.org/become-a-reviewer/
}

(3.9/5), research data information like swMATH (3.2/5) or institution codes (3.5/5) to areas like full-text formula search (3.4/5), where technology is still under development.

Finally, zbMATH offers many freely accessible features. Recently, free author and journal profiles have been added to the traditional three free hits, as well as free EMS member accounts, the swMATH facet and formula search. It is perhaps not surprising that all of these have been quite well received (with marks from 3.6/5 to 4.2/5) and several comments suggest that further steps would be welcomed in this direction. While such efforts are still limited by the need to maintain the resources for zbMATH production and development, we can promise to pursue all feasible solutions. This may also be illustrated by a free referencing tool recently made available to MathOverflow users (with hopefully more to come).

Overall, the survey supports the statement that the SCUC has accompanied a decade of exciting developments for zbMATH. It was decided at the last CC meeting that the duties of the SCUC will, in future, be transferred to the newly formed EMS Committee for Publications and Electronic Disseminations. We would like to take this occasion to thank all the SCUC members for their valuable contributions over the years!

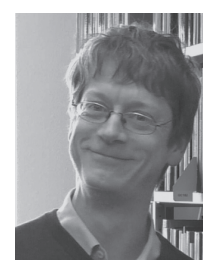

Ingo Brüggemann (Ingo.Brueggemann@ mis.mpg.de) is head librarian of the Max Planck Institute for Mathematics in the Sciences, and served in this function during two terms in the SCUC of zbMATH, bringing into it a certain complementary viewpoint of an in-between. In his role of a Vice-Chair, he coordinated the EMS survey on behalf of the SCUC. His favourite zbMath feature, by the way, is the formula search: math texts are the most formalized of the sciences and this kind of search hints at a way to get a grip on really making the texts' contents machine readable.

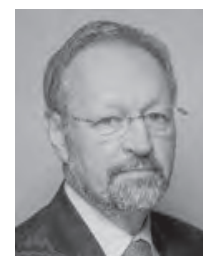

Klaus Hulek studied mathematics at the University of Munich and the University of Oxford. He completed his PhD in Erlangen in 1979 and spent the academic year 1982/83 at Brown University, Providence, RI, USA. He received his habilitation in Erlangen in 1984. Klaus Hulek was a professor of mathematics in Bayreuth from 1985 to 1990, when he moved to Hanover. He held the position of Vice-President of Research of Leibniz Universität Hannover from 2005 to 2014, and from 2007 to 2014 he represented the German Rectors' Conference (HRK) at the Research Policy Working Group of the European University Association (EUA). Klaus Hulek was a member of the Institute for Advanced Study (IAS) in Princeton in 2015. His field of research is algebraic geometry.

Olaf Teschke [olaf.teschke@fiz-karlsruhe.de] is a member of the Editorial Board of the EMS Newsletter, responsible for the zbMATH Column. 